\title{
Sensors based on recycled optical fibers destroyed by the catastrophic fuse effect
}

\author{
Paulo S. André ${ }^{1, *}$, M. Fátima Domingues ${ }^{2}$, Paulo Antunes ${ }^{2}$, Nélia Alberto ${ }^{3}$, Ana Rita Frias ${ }^{2,4}$, R. A. \\ S. Ferreira ${ }^{4}$ \\ ${ }^{1}$ Instituto de Telecomunicações and Department of Electronic and Computer Engineering, Instituto \\ Superior Técnico, University of Lisbon, 1049-001 Lisboa, Portugal \\ ${ }^{2}$ Instituto de Telecomunicações and Departamento de Física da Universidade de Aveiro, 3810-193 \\ Aveiro, Portugal \\ ${ }^{3}$ Instituto de Telecomunicações and TEMA - NRD, Mechanical Engineering Department and Aveiro \\ Institute of Nanotechnology, University of Aveiro, 3810-193 Aveiro, Portugal \\ ${ }^{4}$ Physics Department and CICECO Universidade de Aveiro, 3810-193 Aveiro, Portugal
}

\begin{abstract}
In the last decades the fiber Bragg gratings (FBG) and Fabry-Perot Interferometer (FPI) micro cavities based sensors have become one of the most attractive optical fiber sensing technologies. However, its production requires a significant economical investment. We propose a cost effective solution based on micro cavity generated by the recycling of optical fibers destroyed through the catastrophic fuse effect. This technique considerably reduces the experimental complexity and the production costs. In this paper, the application of these sensors in the monitoring of several parameters, such as refractive index, pressure, strain and temperature is presented.
\end{abstract}

Keyword list: Optical fiber sensor, fiber fuse effect, refraction index, pressure, strain, temperature.

\section{INTRODUCTION}

Nowadays, as result of its advantages over traditional electronic sensing [1], optical fiber sensors are widely used for several applications concerning parameters such as strain and pressure, high temperature and refractive index (RI) sensing, among many others [2-4]. The most suitable optical fiber sensing technologies are based on fiber Bragg gratings (FBG) or Fabry-Perot Interferometer (FPI) micro cavities, due to its small size enclosing an accurate response and sensitivity $[1-3,5,6]$. Nevertheless, the production of such sensing devices demands an high economical investments and requires complex implementations $[7,8]$.

In this work we propose an innovative and cost effective solution to obtain optical fibers FPI sensors, based on micro cavity produced by the recycling of optical fibers destroyed through the catastrophic fuse effect. This technique considerably reduces the process manufacturing costs, making the manufacturing apparatus affordable and requiring small experimental complexity.

\section{SENSORS DESCRIPTION}

The catastrophic fiber fuse effect phenomenon, first observed in 1987, is characterized by the continuous self-destruction of the fiber, by vaporizing the fiber core $[9,10]$. This process is commonly ignited by a local heating point, induced by a high density of energy on a tight bend in a fiber, a damaged or unclean connector. The self-induced destruction is described as fuse zone, propagating towards the optical signal source with an average velocity of $0.5 \mathrm{~m} \mathrm{~s}^{-1}$ [11]. The demand for higher bandwidths on modern optical communications has significantly increased the amount of energy propagating on the fiber network, promoting the catastrophic fuse effect as a real problem on future optical communications networks.

*paulo.andre@lx.it.pt; phone +351218418 486; fax +351218418472

Second International Conference on Applications of Optics and Photonics, edited by Manuel Filipe P. C. Martins Costa, Rogério Nunes Nogueira, Proc. of SPIE Vol. 9286,

$92862 \mathrm{U} \cdot$ (C) $2014 \mathrm{SPIE} \cdot \mathrm{CCC}$ code: 0277-786X/14/\$18 - doi: 10.1117/12.2060191 
The fibers destroyed by this effect presents periodic voids in the core, with dimensions and spatial period in the order of a few micrometers [12]. These voids are in micro cavities, as observed in the microscopic image of Figure 1.a).

To obtain the micro cavity based sensing systems, the destroyed fiber containing the voids was spliced to a standard single mode fiber (SMF) using a splice machine (Fujikura, Model FSM-060S), creating a bigger voids, as seen in Figure 1.b).

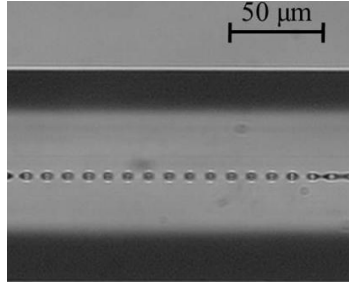

a)

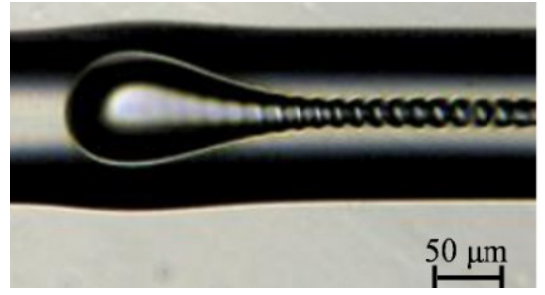

b)

Figure 1. Microscopic images of: a) voids created by the fiber fuse effect and b) the splice of a standard SMF with a destroyed fiber.

To complete the production of the RI and pressure sensing systems, the fiber is cleaved some microns after this bigger void obtaining structures as the ones displayed in Figures 2.a) and 2.b). To obtain micro cavities for the high temperature (up to $1000^{\circ} \mathrm{C}$ ) and strain monitoring, a standard single mode fiber was spliced again to a fiber containing voids as the ones in Figure 2.a) and 2.b), generating the final sensing micro cavities showed in the Figures 2.c) and 2.d).

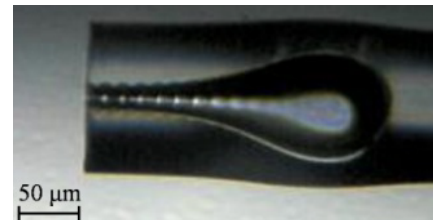

a)

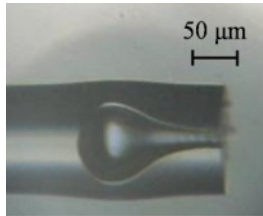

b)

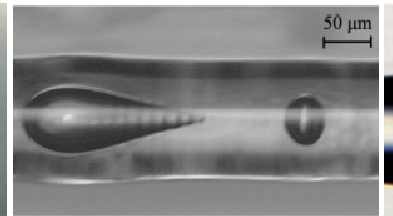

c)

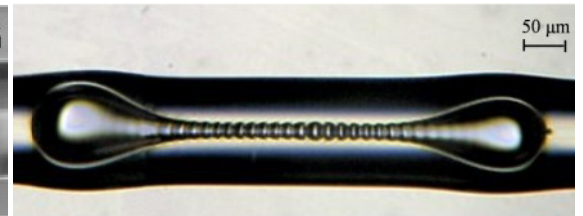

d)

Figure 2. Microscopic images of the micro cavities produced for the sensing of: a) RI; b) pressure; c) strain; d) high temperature.

Considering the traditional micro cavities fabrication methods, based on femtosecond lasers $[5,6]$, this technique considerably reduces the manufacturing costs and the complexity of the process.

\section{EXPERIMENTAL IMPLEMENTATION AND RESULTS}

An FPI is typically composed by two reflecting surfaces with an intermediate gap, creating a periodic reflection transfer function in the spectral frequency domain. The optical fiber micro cavities produced behave as a FPI, whose dimensions impacts on the reflected optical spectrum characteristics and so, the optical phase difference $\left(\phi_{F P I}\right)$, according with the following equation:

$$
\emptyset_{F P I}=\frac{4 \pi}{\lambda} n L
$$

where $\lambda$ is the signal wavelength, $n$ is the cavity material refractive index (here considered as air, $\mathrm{n}=1$ ) and $L$ is the cavity physical length. This relation, applicable on FPI cavities will be used to characterize the sensors produced.

\subsection{Refractive index sensor}

The produced RI sensor (Figure 2.a)) has a cavity length of $251.7 \pm 1.0 \mu \mathrm{m}$, a maximum width of $89.1 \pm 0.7 \mu \mathrm{m}$ and a width in the fiber tip of $18.8 \pm 0.7 \mu \mathrm{m}$. In this cavity, the reflection transfer function is periodic in the frequency domain, with a period that depends on the surrounding environment refractive index, according with the equation (2). This equation describes the relation between the modulation period, $\Gamma$, the signal wavelength, $\lambda_{0}$, the cavity effective refractive index, $n_{\text {eff }}$ and the cavity length, $L$ : 


$$
\Gamma=\frac{\lambda_{0}^{2}}{2 n_{e f f^{L}}}
$$

To investigate the RI sensing performance, the sensor was tested using aqueous solutions with different saccharose concentrations, which provide a RI variation ranging from 1.3320 and 1.4281 , measured using a $8^{\circ}$ tilted fiber Bragg grating based refractometer [13]. In order to guarantee a constant cavity length $(L)$, the experimental tests were performed in a controlled environment of constant pressure and temperature.

The manufactured sensor sensitivity to RI changes was estimated by immersing the optical fiber end tip in the saccharose solutions, Figure 3. A fiber support allows the control, and keeping constant, the depth at which the sensor head is immersed in the aqueous solutions.

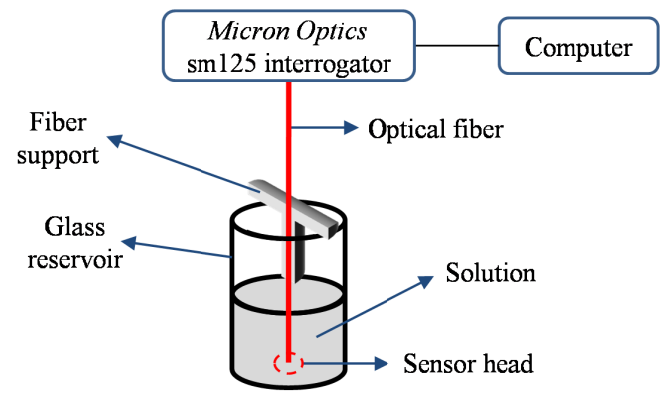

Figure 3. Experimental setup for the RI sensor test.

The reflected spectra were monitored using an optical sensing interrogator, model sm125 from Micron Optics, with 1 pm resolution. Figure 4 shows the sensor reflection spectra under immersion, showing a frequency decrease in the reflected spectrum modulation as the solution RI increased, which is in agreement with behavior predicted by equation (2). The increase of the solution refractive index results in the increase of the refractive contrast (to the cavity medium refractive index $\approx 1$ ) and therefore in the increase of the reflection coefficient and consequently of the modulation depth. The modulation period was measured, using the peak selection method over a wide spectral region, as function of the RI. In a close analysis, it becomes clear that the experimental data reveals a nearly linear dependence in the range of refractive indices from 1.3320 to 1.4281 (the range tested), Figure 4.b). From its linear fit, the sensor sensitivity was estimated, yielding to a value of $-1.81 \pm 0.20 \mathrm{~nm} / \mathrm{RIU}$.

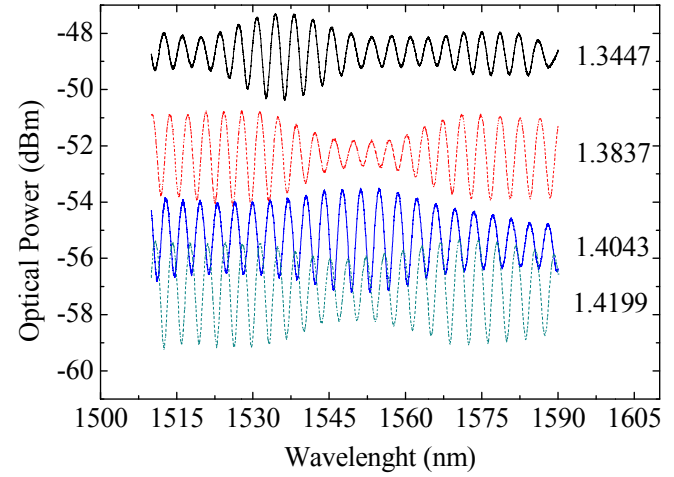

a) b)

Figure 4.a) Micro cavity optical reflection spectra measured for the different saccharose aqueous solutions (RI values are shown on the right side of the spectra, measured with a RFBG sensor). b) Micro cavity optical reflection spectra wavelength modulation period as function of the refractive index of the solutions tested (the line represents the linear fit to the experimental data, $r>0.93$ ).

Considering the resolution of the optical interrogation system $(1 \mathrm{pm})$, a resolution of $5.5 \times 10^{-4}$ RIU was estimated for the sensor proposed. These values for the modulation period and modulation period sensitivity are in agreement with the estimations obtained from the theoretical model used to describe the micro cavity based sensor. 


\subsection{Pressure sensor}

The FPI micro cavities approach for pressure sensing has a similar setup as the one used for RI sensing, with the sensor dimensions of $169.8 \pm 2.3 \mu \mathrm{m}$ for the cavity length, a maximum width of $91.8 \pm 0.9 \mu \mathrm{m}$ and a width in the fiber tip of $21.2 \pm 0.6 \mu \mathrm{m}$. However, in this particular situation, the sensibility to pressure was tested by placing the sensor inside a recipient, and gradually increasing the water column high above it in the recipient in $4 \mathrm{~cm}$ steps, inducing the cavity length $L$ to decrease. The typical wavelength spectra obtained for this tests are displayed in Figure 6.a), and the wavelength modulation period as function of the pressure induced in the sensor for the water levels in analysis is displayed in Figure 6.b).

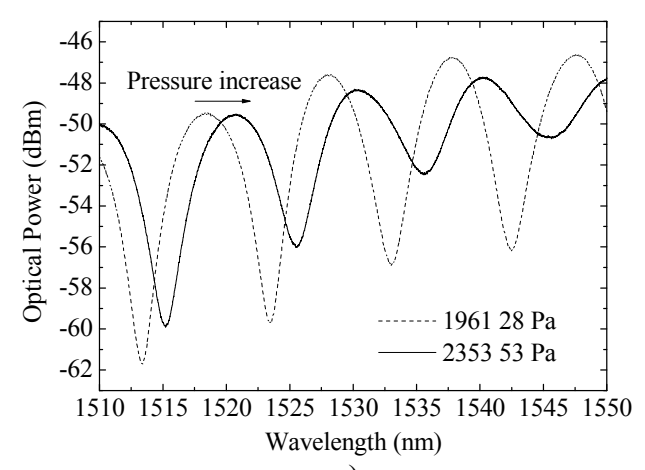

a)

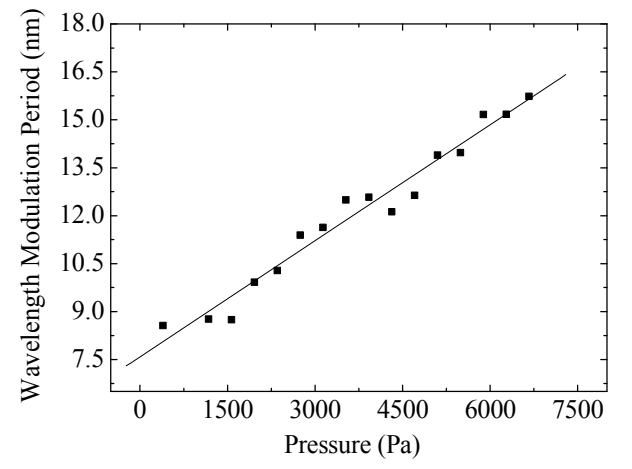

b)

Figure 5. a) Micro cavity reflection spectra for different values of applied pressure; b) Micro cavity modulation period as function of increase pressure (the line corresponds the best fit to the experimental data, $r>0.96$ )

From the experimental data analyzed a sensitivity of $1.21 \pm 0.06 \mathrm{pm} / \mathrm{Pa}$ was achieved, which reveals an enhancement in the sensitivity when compared with similar sensors manufactured through more demanding complex experimental apparatus [14].

\subsection{Strain sensor}

For the analysis of the micro cavities for strain sensing, a micrometric translation stage was used. The fiber holding the micro cavity (Figure 2.c), with the dimensions of $41.5 \pm 0.5 \mu \mathrm{m}$ in the longitudinal axis and $55.3 \pm 0.5 \mu \mathrm{m}$ in the transversal axis) was fixed between a rigid fixed support and the linear translation stage, with a distance between anchorage points of $13.0 \mathrm{~cm}$. The sensor reflection spectrum was obtained as function of the imposed elongation, ranging from $0 \mu \mathrm{m}$ to $750 \mu \mathrm{m}$, which corresponds to a maximum strain value of $5769 \mu \varepsilon$. The reflected optical spectra for different values of the applied strain are shown in Figure 6.a), and the strain sensitivity, obtained by the linear fit of the spectral wavelength shift as a function of the applied strain, is estimated in Figure 6.b).

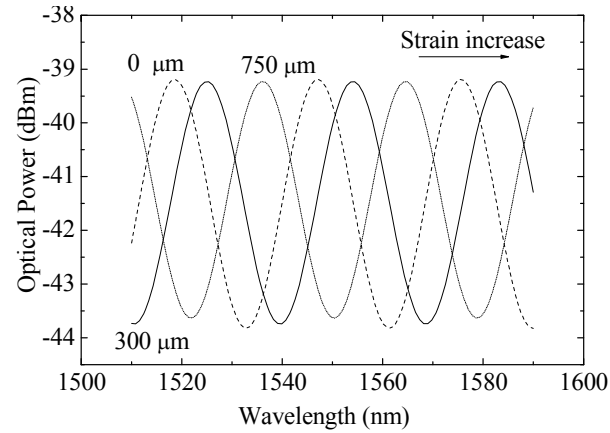

a)

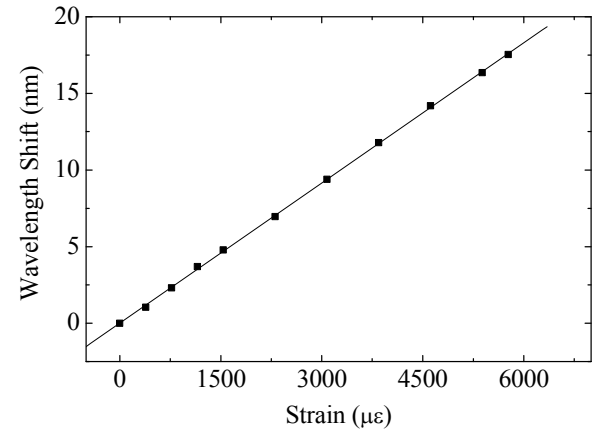

b)

Figure 6. a) Measured reflection optical spectra for different strain values; b) Spectral wavelength shift as function of the applied strain. The dots represent the experimental data and the lines are the best linear fits $(r>0.99)$.

This characterization was performed until the fibers fracture, revealing a rupture limit of $5769.23 \mu \varepsilon$, and a strain sensitivity of $3.1 \pm 0.1 \mathrm{pm} / \mu \varepsilon$. These values are identical, within the experimental uncertainty, to those obtained for the rupture limit of single mode silica optical fibers [15], pointing out that the this micro cavities production process does not debilitate the optical fiber robustness. 
A cavity under a physical perturbation will undergo a physical length change. Therefore, a longitudinal strain applied to the FPI optical cavity will change the cavity physical length, resulting in the wavelength shift of the FPI cavity reflection transfer function. The wavelength shift can be related with the longitudinal relative elongation, $\varepsilon_{z}$, by [16]:

$$
\frac{d \lambda_{m}}{d z}=\frac{4}{(2 m+1)} L \varepsilon_{\mathrm{z}}
$$

where $m$ is the order of the interference. Although equation (4) shows an explicit dependence with the cavity physical length, the $m$ parameter is also dependent on $L$, as shown before in [16] and a fact often ignored by other authors [5,17]. When a strain is applied along the longitudinal axis of the fiber, it will also induce a reduction of the cavity with, due to the small diameter of the fiber. This effect make the micro cavity response to external deformation (strain sensitivity)

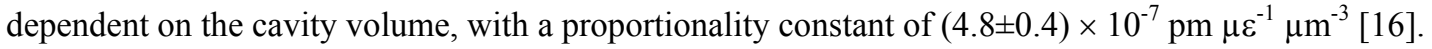

\subsection{High temperature sensor}

For the thermal characterization the micro cavity based sensor was placed in a tubular furnace, and the temperature was increased from $22^{\circ} \mathrm{C}$ till $1150{ }^{\circ} \mathrm{C}$ in $100^{\circ} \mathrm{C}$ steps. Figure 7 shows the reflected optical spectrum and the spectrum wavelength shift as function of the temperature values.

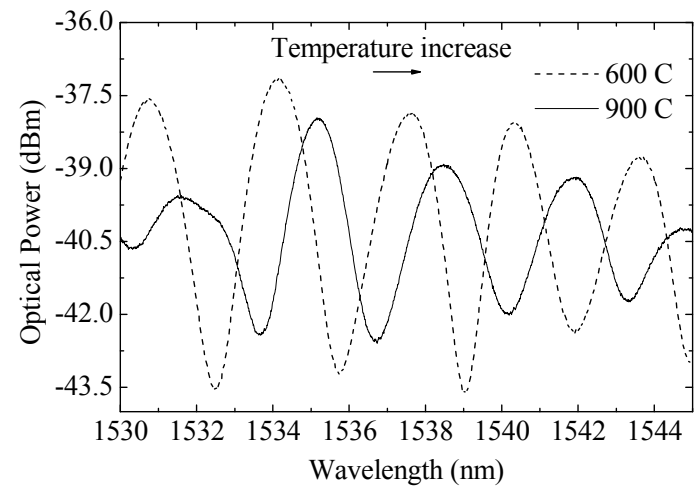

a)

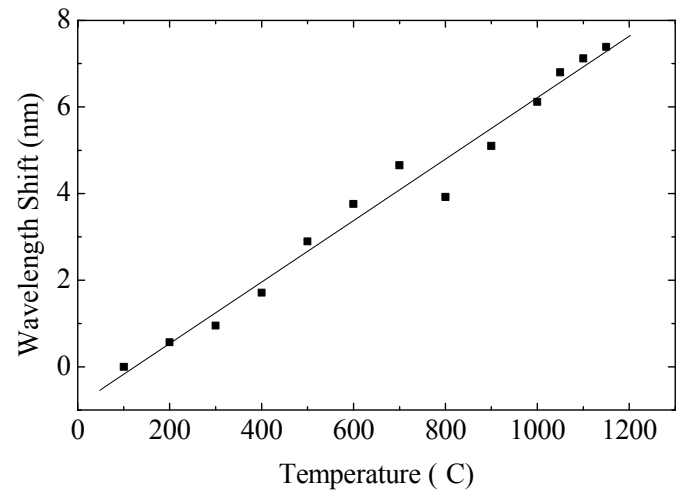

b)

Figure 7. a) Spectral response for two different temperatures, and b) Spectral wavelength shift as function of the temperature increase. The dots represent the experimental data and the lines are the best linear fits $(r>0.98)$.

Considering the influence of the temperature, $T$, in the micro cavity length (with an initial value of $626.4 \pm 2.3 \mu \mathrm{m}$ ), a sensitivity of $7.1 \pm 0.3 \mathrm{pm} /{ }^{\circ} \mathrm{C}$ was achieved with stable responses temperatures up to $1150{ }^{\circ} \mathrm{C}$. The value obtained for this sensor is close to the sensitivity obtained for typical uniform FBG device, $13.9 \pm 0.3 \mathrm{pm} /{ }^{\circ} \mathrm{C}$, with the advantage that these micro cavities sensors were able to stand temperatures up till $1150{ }^{\circ} \mathrm{C}$, while a FBG sensor is erased at temperatures above $500{ }^{\circ} \mathrm{C}$. Additionally, the micro cavities based sensors stability at $1000{ }^{\circ} \mathrm{C}$ was tested, showing its ability to stand such harsh conditions for several hours, with no significant changes in the reflected spectra.

When compared with similar high temperature sensors, like RFBG $[18,19]$, the micro cavities sensor present similar robustness and a sensitivity in the same order of magnitude as a typical RFBG $\left(16.1 \pm 0.1 \mathrm{pm} /{ }^{\circ} \mathrm{C}\right)$ with a low cost and less complex manufacturing setup.

\section{CONCLUSION}

In this work we present an innovative and cost effective process for FPI micro cavities sensing systems through the recycling of optical fiber destroyed by the catastrophic fuse effect, with applications in RI, pressure, strain and high temperature measurements. The sensitivities obtained for the developed devices are generally in the range of similar systems produced through high cost experimental apparatus which makes the solution reported an advantage regarding the low cost and complexity of production and implementation. 


\section{ACKNOWLEDGMENTS}

This work was supported in part by Fundação para a Ciência e Tecnologia (FCT) through the Ph.D. fellowship SFRH/BD/69097/2010 and the Post-doctoral fellowships SFRH/BPD/78141/2011 and SFRH/BPD/76735/2011. FEDER, COMPETE are also acknowledged (LA0011/2013/CICECO-FCOMP-01-0124-FEDER-037271 and PEstOE/EEI/LA0008/2013).

\section{REFERENCES}

[1] Lopez-Higuera, J. M., Rodriguez Cobo, L., Quintela Incera, A., and Cobo, A., "Fiber Optic Sensors in Structural Health Monitoring,” J. Lightw. Technol., Journal of, 29, 587-608 (2011).

[2] Basumallick, N., Chatterjee, I., Biswas, P., Dasgupta, K., and Bandyopadhyay, S., "Fiber Bragg grating accelerometer with enhanced sensitivity," Sensor. Actuat. A- Phys., 173, 108-115 (2012).

[3] Leitão, C., Bilro, L., Alberto, N., Antunes, P., Lima, H., André, P. S., Nogueira, R., and Pinto, J. L., "Feasibility studies of Bragg probe for noninvasive carotid pulse waveform assessment," J. Biomed. Opt., vol. 18, pp. 017006017006, 2013.

[4] Mishra, V., Singh, N., Tiwari, U., and Kapur, P., "Fiber grating sensors in medicine: Current and emerging applications," Sensor. Actuat. A- Phys, 167, 279-290 (2011).

[5] Liao, C. R., Hu, T. Y., and Wang, D. N., "Optical fiber Fabry-Perot interferometer cavity fabricated by femtosecond laser micromachining and fusion splicing for refractive index sensing," Opt. Express, 20, 2281322818 (2012).

[6] Ran, Z., Li, C., Zuo, H., and Chen, Y., "Laser-Machined Cascaded Micro Cavities for Simultaneous Measurement of Dual Parameters Under High Temperature,” Sensors J., IEEE, 13(5), 1988-1991 (2013).

[7] Lee, B. H., Y. Kim, H., Park, K. S., Eom, J. B., Kim, M. J., Rho, B. S., and Choi, H. Y., "Interferometric Fiber Optic Sensors," Sensors, 12, 2467-2486 (2012).

[8] Zhu, T., Wu, D., Liu, M., and Duan, D.W., "In-Line Fiber Optic Interferometric Sensors in Single-Mode Fibers," Sensors, 12, 0430-10449 (2012).

[9] Kashyap, R. and Blow, K. J., "Observation of Catastrophic Self-Propelled Self-Focusing in Optical Fibers," Electron. Lett., 24, 47-49 (1988).

[10] Domingues, F., Rocha, A. M., and Andre, P. S., "High-Power Effects in Damaged and Contaminated Optical Fiber Connectors," Microw. Opt. Techn. Let., 53, 2485-2488 (2011).

[11] Rocha, A. M., Antunes, P. F. C., Domingues, F., Facão, M., and André, P., "Detection of Fiber Fuse Effect Using FBG Sensors, 2 Sensors J., IEEE, 11, 1390-1394 (2011).

[12] Domingues, F., Frias, A. R., Antunes, P., Sousa, A. O. P., Ferreira, R. A. S., and Andre, P. S., "Observation of fuse effect discharge zone nonlinear velocity regime in erbium-doped fibres," Electron. Lett., 48, 1295-1296 (2012).

[13] Alberto, N., Marques, C., Pinto, J. L. and Nogueira, R., "Three-parameter optical fiber sensor based on a tilted fiber Bragg grating," Appl. Optics, 49, 6085-6091 (2010).

[14] Zhang, Y., Yuan, L., Lan, X., Kaur, A., Huang, J., and Xiao, H., "High-temperature fiber-optic Fabry Perot interferometric pressure sensor fabricated by femtosecond laser," Opt. Lett. 38, 4609-4612 (2013).

[15] Antunes, P., Lima, H., Monteiro, J., and André, P. S., "Elastic constant measurement for standard and photosensitive single mode optical fibres," Microw. Opt. Techn. Let, 50, 2467-2469 (2008).

[16] Antunes, P.F.C., Domingues, M.F.F., Alberto, N.J., Andre, P.S., 2Optical Fiber Microcavity Strain Sensors Produced by the Catastrophic Fuse Effect," Photon. Technol. Lett., IEEE , 26(1), 78-81 (2014).

[17] Favero, F. C., Araujo, L., Bouwmans, G., Finazzi, V., Villatoro, J., and Pruneri, V., "Spheroidal Fabry-Perot microcavities in optical fibers for high-sensitivity sensing," Opt. Express, 20, $7112-7118$ (2012).

[18] Alberto, N. J., Santos, J. A., Marques, C. A. F., Neto, V. F. S., Nogueira, R. N., "Nanodiamond coated Bragg gratings for sensing applications," Proc. SPIE 8421, OFS2012 22nd International Conference on Optical Fiber Sensors (2012).

[19] Alberto, N. J., Simões, R., Oliveira, V., Kalinowski, H. K., Neto, V. F., Nogueira, R. N., "Thermal monitoring of the diamond deposition process using regenerated FBG," Proc. SPIE 8794, Fifth European Workshop on Optical Fibre Sensors (2013). 